\title{
The Research on Distribution of Benefits in the Cooperation of Enterprises, Colleges and Institutes Based on Shapley Model
}

\author{
Zhan meng-shi ${ }^{1,}{ }^{*}$, Cai qi-ming ${ }^{1}$, Liu shuang-teng ${ }^{1}$ \\ ${ }^{1}$ College of Economics and Management, Nanjing University of Aeronautics and Astronautics, \\ Nanjing 210016, China \\ *email: 1033329568@qq.com
}

\begin{abstract}
Based on the analysis of influence factors of distribution of benefits in the collaborative innovation of enterprises, colleges and institutes, improved Shapley value is proposed based on the revised intervals, the research collaborative innovation research interests allocation problem , and the distribution of benefits were studied by using Shapley value model. Firstly, Shapley value model was described and analyzed in this paper. Then according to the characteristics of collaborative innovation of enterprises, colleges and institutes, and union bargaining power, project risk factors, project completion, etc. were evaluated by introducing intervals, and penalty function and excitation function were established to correct the Shapley value model .Ultimately through a case study, it can confirm that the Shapley value model based on the revised intervals established in this paper is effective and fair.
\end{abstract}

Keywords: enterprises, colleges and institutes distribution of benefits Shapley value model intervals excitation function.

\section{Introduction}

With the increasingly fierce market competition and the accelerating pace of updating the knowledge, seeking innovative enterprises in the development of the road began to seek research institutes and universities to collaborative innovation ${ }^{[1]}$. China in 2011 officially launched the implementation of "Plan 2011", led by a college, the government, enterprises and others to resources sharing, complementary advantages and establishment of collaborative innovation centers. The research collaborative innovation distribution of interests is the key of enterprises, universities and research institutes to cooperate or not.

1953 Shapley and others made Shapley value method is a good way to solve a multiplayer cooperative game in allocation of interest ${ }^{[2-3]}$. But suppose establish equal bargaining power, which does not match with the actual situation is relatively cooperative game average on the basis of no risk. To this end, Shapley and others considering randomness, technological innovation, and other risk factors on the basis of the traditional Shapley value model for continuous improvement ${ }^{[4-6]}$. But these improvements Shapley models assume various influencing factors are weighted equally, it is not well in line with the actual situation.

$\mathrm{Na} \mathrm{Xu}$, who through venture investment, information disclosure, and failed to consider the Fourth and supply chain alliance to Shapley model was modified, but does not consider the factors leading enterprises ${ }^{[7]}$; Haeringer Per capita raised more weight Shapley value model, modified to reflect the model, although the members of the alliance in the right weight is different, but for the weight vector obtained does not clearly set forth ${ }^{[8-9]}$. O Palanc1 considerations and future potential of close cooperation in the collaborative game factor, the introduction of the concept of interval gray number, gray Shapley value is proposed model ${ }^{[10]}$; Zhang Yanfeng, who also proposed a model based on Shapley risk factor correction, considering conditions for cooperation partners for analysis [11-12]. These Shapley model based on the weight of the modified reflecting league different weights, but does not reflect the weighting set is reasonable, the lack of a realistic model of rational evaluation.

With the introduction of the concept and development of research cooperation, the income distribution problem also attracted the attention of more and more scholars and research. Lee first proposed in research alliances, there are a lot of tacit knowledge transfer can not be shared by economic indicators to accurately measure ${ }^{[13]}$. Huang Bo through the Cooperative interest 
distribution is analyzed, and the optimal distribution of benefits under the scheme in different ways depending on their excitation efficiency [14]. Zhan Meiqiu etc are using different game model further analysis of the distribution of the benefits of research cooperation ${ }^{[15-16]}$. Then, there are the interests of Distribution of research scholars used Shapley model method, Shapley value and improve the model. Li Bozhou, etc using the Shapley Value intermediaries will be incorporated into the distribution of benefits of research cooperation and to establish a model based on the Shapley risk factor correction ${ }^{[17]}$.

The above methods of Shapley value model is improved, but the practical application of research collaborative innovation lack of consideration for the members of the Union bargaining power and process evaluation, the lack of suitable comprehensive evaluation and revision of earnings hairs, while research cooperation regarded as one-time, so there are some non-adaptive. From the above discussion, we introduce amendments interval number modeled and analyzed, computing and other instances.

All manuscripts must be in English. Please keep a second copy of your manuscript in your office (just in case anything gets lost in the mail). When receiving the manuscript, we assume that the corresponding authors grant us the copyright to use the manuscript for the book or journal in question. Should authors use tables or figures from other Publications, they must ask the corresponding publishers to grant them the right to publish this material in their paper. Use italic for emphasizing a word or phrase. Do not use boldface typing or capital letters except for section headings (cf. remarks on section headings, below). Use a laser printer, not a matrix dot printer.

\section{Shapley Value of The Original Model}

\subsection{Basic Assumptions}

Shapley value Shapley LS model is proposed to solve the problem of many men cooperation countermeasure a mathematical method ${ }^{[18]}$. Shapley value method has two basic assumptions: First, when members to cooperate, arbitrary number of members of the cooperative among them will cause each member to obtain a certain income; the second is when the $n$ member alliance, the alliance the total revenue will be greater than $n$ members individually complete the proceeds.

\subsection{Model Representation}

$\mathrm{N}=\{1,2 、 \cdots i \cdots n\}$ finite set for a given $n$ people constituted, $\mathrm{N}$ in any subset $S$ (any of cooperation in humans) corresponds to a real-valued function $V(S)$, the following conditions are met:

$$
\begin{gathered}
V(\phi)=0, \phi \text { is empty set. } \\
V\left(S_{1} \cup S_{2}\right) \geq V\left(S_{1}\right)+V\left(S_{2}\right), S_{1} \cap S_{2}=\phi
\end{gathered}
$$

$V(S)$, represents the maximum benefit from $S$ through alliances available to participants, saying that $(V, N)$ is $N$ an $n$ person Cooperation.

For any $S_{1}, S_{2}$ if $V\left(S_{1} \cup S_{2}\right) \geq V\left(S_{1}\right)+V\left(S_{2}\right), S_{1} \cap S_{2}=\phi, V$ is called super-additive countermeasures. Meanwhile, Superadditive Countermeasures meet the following criteria:

$$
\begin{aligned}
& \sum_{i=1}^{n} x_{i}=V(N) \quad i=1,2, \ldots, n \\
& x_{i} \geq V(i) \quad i=1,2, \ldots, n
\end{aligned}
$$

Wherein, $x_{i}$ represents $i$ in revenue from members of the alliance should get benefits include $\mathrm{N}$, $x=\left(x_{1}, x_{2}, \ldots x_{n}\right)$ represents Countermeasures cooperation.

In the league, the income obtained by Shapley value method called Shapley value of each participant's participants, referred to as $\phi(\mathrm{v})=\left(\phi_{1}(\mathrm{v}), \phi_{2}(\mathrm{v}) \cdots \phi_{\mathrm{i}}(\mathrm{v}) \cdots, \phi_{\mathrm{n}}(\mathrm{v})\right)$, Y represents earnings cooperation with the members of the Shapley Value obtained obtained.

In the league, the income obtained by Shapley value method called Shapley value of each participant's participants, referred to as $\phi(\mathrm{v})=\left(\phi_{1}(\mathrm{v}), \phi_{2}(\mathrm{v}) \cdots \phi_{\mathrm{i}}(\mathrm{v}) \cdots, \phi_{\mathrm{n}}(\mathrm{v})\right) . \phi_{\mathrm{i}}(\mathrm{v})$ represents income obtained by Shapley Value in cooperation $n$ member obtained in $i$. 


$$
\begin{gathered}
\phi_{i}(v)=\sum_{s \subset s_{i}} W(s)[v(s)-v(s / i)] \\
w(s)=\frac{(n-s) !(s-1) !}{n !} \quad s=\left|S_{\mathrm{i}}\right|
\end{gathered}
$$

Wherein, $s$ is the number of participants in a subset of $S, w(s)$ is the weighting factor, $v(s / i)$ for removing efficiency $f$ e obtained by the members. However, with model description can be found, there is a Shapley Value absolutely fair distribution of benefits and other issues ${ }^{[19-21]}$.

\section{Interval Shapley Value Model Based on The Number of Amendments}

\subsection{Description Model Updating}

Shapley value allocation method does not consider the importance of the members, the difficulty of the project, performance evaluation and other factors, so this distribution exhibits fairness is actually an average of establishing fairness on, so its solving practical problems meaning when compromised. In addition, research cooperation, the enterprise bargaining power is higher than most universities and research institutes.so, in a dominant position of enterprises will consider seeking potential long-term cooperation, sacrifice their own short-term interests, give more benefits of space science research party. Thus, Shapley value method of distribution of interests in the new environment will be the presence of certain non-adaptive ${ }^{[22-23]}$.

Thus, the traditional Shapley value distribution model is applied research in the interests of cooperation, its lack of respect for the members of the importance of factors and lack of bargaining power and other considerations will be more prominent, it exacerbated the inequities alliance practical sense. To this end, the paper Shapley value model was improved by introducing interval number of this tool, the establishment of penalties, development and reward three evaluation period, and to determine the punishment, development, activation function, to achieve the target object evaluation and examination.

Shapley value for the modified model is described as follows:

1: Corporate Universities or research institutes a comprehensive evaluation and examination, the main factors taken into account in accordance with the impact on the production side factors were divided into positive and negative factors. For example, the trust factor is higher, the main square for the better, the higher the evaluation value, positive factors; the higher paid capital of this factor, the main square facing the risk, the greater its more unfavorable Reviews a lower value for the negative factors.

2: Because of the influence factors when evaluating the ambiguity and uncertainty, for the introduction of the concept of interval number. But for each of the evaluation factors influence the maximum value, minimum value and the difference between the evaluation period must be the same. Among them, various factors affect the evaluation of specific gravity can be obtained by AHP method.

\subsection{Setting punishment incentive zone}

Improved optimization model is divided into two phases:

The first stage is to evaluate each factor, according to the weights aggregated.

Suppose the positive factors as $x_{k}$, negative factors for the $y_{k}, k \in(1, n)$. Factors affecting the right of any corresponding weight $x_{k}$ right to $\alpha_{k}, y_{k}$ corresponding to the weight of $\beta_{k}$, then:

$$
\alpha_{1}+\alpha_{2}+\ldots . . \alpha_{n}+\beta_{1}+\beta_{2}+\ldots . . \beta_{n}=1
$$

Positive and negative factors $x_{k}$ and $y_{k}$ are evaluated scoring, score intervals were $\left[a_{1}, b_{1}\right],\left[a_{2}, b_{2}\right], \ldots,\left[a_{n}, b_{n}\right],\left[c_{1}, d_{1}\right],\left[c_{2}, d_{2}\right], \ldots,\left[c_{n}, d_{n}\right]$, any of its influencing factors in the evaluation scores are between $\left[m_{0}, n_{0}\right]$, and scores range span equal. The evaluation function Gakken parties as follows:

$$
G_{i}=\alpha_{1}\left[a_{1}, b_{1}\right]+\alpha_{2}\left[a_{2}, b_{2}\right]+\ldots \ldots+\alpha_{n}\left[a_{n}, b_{n}\right]+\beta_{1}\left[c_{1}, d_{1}\right]+\beta_{2}\left[c_{2}, d_{2}\right]+\ldots . .+\beta_{n}\left[c_{n}, d_{n}\right]
$$


So $G_{i}=[m, n]$, and $G_{i} \in\left[m_{0}, n_{0}\right]$.

The second phase will be based on Gakken party evaluation to determine the punishment zone, development zone, excitation zone.

The number line interval $\left[m_{0}, n_{0}\right]$ by numerical size divided into three areas, the penalty areas, development areas, incentive zone. $\left[m_{0}, n_{0}\right]$ is the midpoint of the interval $\frac{m_{0}+n_{0}}{2}$, take $2 \omega \%$ throughout the mid-range as a development area $(\omega \in(0,50))$, then:

development zone is: $\left[\frac{m_{0}+n_{0}}{2}-\omega \%\left(n_{0}-m_{0}\right), \frac{m_{0}+n_{0}}{2}+\omega \%\left(n_{0}-m_{0}\right)\right]$,

punishment zone is: $\left[m_{0}, \frac{m_{0}+n_{0}}{2}-\omega \%\left(n_{0}-m_{0}\right)\right]$,

excitation zone is: $\left[\frac{m_{0}+n_{0}}{2}+\omega \%\left(n_{0}-m_{0}\right), n_{0}\right]$.

Divide each section shown in Figure 1 :

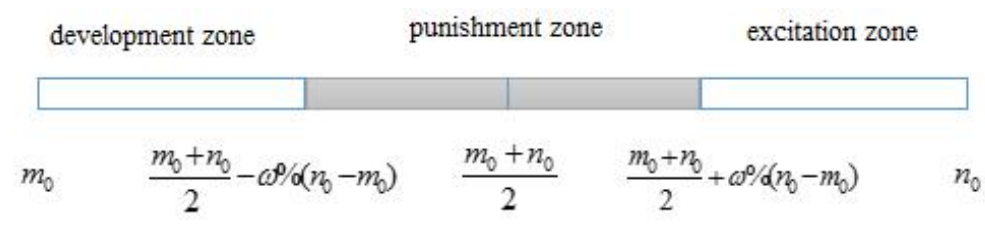

Fig. 1 Punishment develop motivate interval graph

\subsection{The Introduction of Variable Factor Correction}

Since each project has its uniqueness, potential for long-term cooperation projects, production is willing to provide more space in order to encourage long-term cooperation, at the same time the difficulty and risk of the project is also affecting the punishment zone, development zone, incentive size area. For this time interval is divided consider the project as well as project risk factors, added to a variable factor $g(g \in(-50,50))$.

$g$ the size and difficulty of the project, project risk, estimated duration factors. Difficulty g size and duration of the project and proportional to expect that the greater the degree of difficulty of the project and the project is expected at the higher the duration, the smaller the size of g; the larger the size of the project risk is inversely proportional to $\mathrm{g}$, that project risk, $\mathrm{g}$ larger the size. $g$ factor variable size does not change the size of the development area, but the effect of changes in the position of the development zone. After the three regions to join variable factor correction are:

development zone is: $\left[\frac{m_{0}+n_{0}}{2}-(\omega-g) \%\left(n_{0}-m_{0}\right), \frac{m_{0}+n_{0}}{2}+(\omega+g) \%\left(n_{0}-m_{0}\right)\right]$

punishment zone is: $\left[m_{0}, \frac{m_{0}+n_{0}}{2}-(\omega-g) \%\left(n_{0}-m_{0}\right)\right]$

excitation zone is : $\left[\frac{m_{0}+n_{0}}{2}+(\omega+g) \%\left(n_{0}-m_{0}\right), n_{0}\right]$

Divide each section shown in Figure 2 :

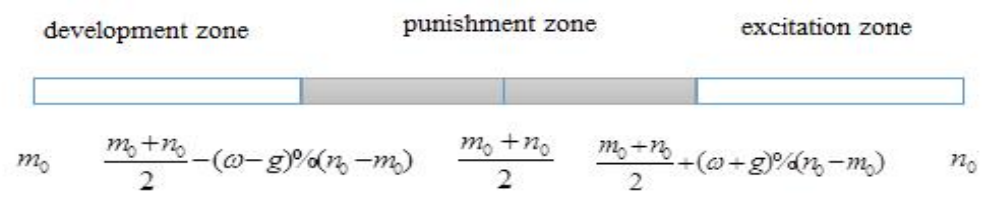

Fig. 2: Punishment, develop, motivate interval graph containing the variable factor Punishment, development, incentive zoning as follows: 
1 ) If the evaluation value Gakken parties located $\left[m_{0}, \frac{m_{0}+n_{0}}{2}-(\omega-g) \%\left(n_{0}-m_{0}\right)\right]$, namely punishment area, suggesting that the overall performance of poor cooperation in research Secondary research side, Gakken square its overall strength is not strong and the overall strength of the contribution of ships or ships with low contribution;

2 ) If the evaluation value Gakken parties located $\left[\frac{m_{0}+n_{0}}{2}-(\omega-g) \%\left(n_{0}-m_{0}\right), \frac{m_{0}+n_{0}}{2}+(\omega+g) \%\left(n_{0}-m_{0}\right)\right]$, namely the development zone, which indicates that the overall performance of high school research cooperation parties are satisfied with Gakken square its overall strength and general contribution of ships or strong comprehensive strength and a lower contribution in research;

3 ) If the evaluation value Gakken parties located $\left[\frac{m_{0}+n_{0}}{2}+(\omega+g) \%\left(n_{0}-m_{0}\right), n_{0}\right]$, namely excitation region, suggesting that the overall performance of high school research cooperation party well in research, Gakken square its overall strength and general contribution or a combination of high strength and high general contribution.

\subsection{Shapley Model Interval Number Amended}

By Gakken party evaluation value partition, you can define the cooperation of industry partners as well as the attitude of the corresponding measures, can also be adjusted corresponding to the distribution of benefits based on different projects and partners. Based on the evaluation value Gakken parties determine their respective section, and set three function penalty function $p(v)$ as punishment zone, development zone, zone incentives, the development of the function $q(v)$, activation function $r(v)$, then the distribution of benefits in the Cooperative Middle School Institute Party becomes:

$$
V_{i}^{\prime}=\phi_{i}(v)+\Delta V_{i}
$$

Where, $V_{i}^{\prime}$ is the total revenue corrected, $\Delta V_{i}$ is the punishment that may arise, develop, motivate combination of the probability function.

If $G_{i} \in\left[m_{0}, \frac{m_{0}+n_{0}}{2}-(\omega-g) \%\left(n_{0}-m_{0}\right)\right]$, namely the evaluation entirely punitive area, the square of Gakken penalty function to determine the probability of return of $100 \%, V_{i}^{\prime}=\phi_{i}(v)+p(v)$;

If $G_{i} \in\left[\frac{m_{0}+n_{0}}{2}-(\omega-g) \%\left(n_{0}-m_{0}\right), \frac{m_{0}+n_{0}}{2}+(\omega+g) \%\left(n_{0}-m_{0}\right)\right]$, namely evaluation is completely attributable to the development area, the use of Gakken party development function to determine the probability of return of $100 \%, V_{i}^{\prime}=\phi_{i}(v)+q(v)$;

If $G_{i} \in\left[\frac{m_{0}+n_{0}}{2}+(\omega+g) \%\left(n_{0}-m_{0}\right), n_{0}\right]$, That evaluation entirely excitation area, the use of Gakken side excitation function to determine the probability of return is $100 \%, V_{i}^{\prime}=\phi_{i}(v)+r(v)$;

If $G_{i}$ simultaneously in the two regions, such as the same time in the development zones and incentives region and their respective percentage of the order of $z$ and $1-z$, then for Gakken party through development function to determine the benefits of probability $z$, for Gakken party adopt incentive function to determine income probability is $1-z$.Probability function can be used in combination to determine its ultimate benefit is $V_{i}^{\prime}=\phi_{i}(v)+z \cdot q(v)+(1-z) r(v)$. 


\section{Case Analysis}

\subsection{Cases Operators}

There are three participants $i=1,2,3$, respectively, universities, research institutes and an enterprise of a certain production technology developed in a research cooperation project. The main project is the production line of new technology research and innovation, enterprise-led, universities and research institutes to participate, including the results of new technology, product line innovations, patents and other achievements. Benefits that can be obtained by combining the participants as follows (Unit: million)

$v(1)=20, v(2)=16, v(3)=18 ; v(1,2)=45, v(1,3)=48, v(2,3)=44 ; v(1,2,3)=70$

By equation (5), (6) to calculate the original Shapley value distribution model of the participating members' interests are: Next, the Shapley value model is modified.

The overall strength of colleges and universities are relatively strong, in cooperation contribution value is relatively high, companies are expected to carry out its long-term cooperation; and some of the strength of the weaker research institutes, the project primarily as an auxiliary university cooperation, not many companies are expected to cooperate with future opportunities. Business-to-1 in a university and a research hospital examination to evaluate two main factors to consider in Table 1 below:

Table 1: Gakken party to evaluate the impact of factors

\begin{tabular}{l|l|l}
\hline \multicolumn{2}{l|}{ Positive factors $\left(x_{k}\right)$} & Negative Factors $\left(y_{k}\right)$ \\
\hline Trust & research ability & Paid in capital \\
\hline Cooperation time & Project completion & Corporate contribution rate \\
\hline Gakken party contribution & Facility size & \\
Factors right weight & $\alpha=\left(\alpha_{1}, \alpha_{2}, \ldots \alpha_{6}\right)=(0.1,0.15,0.1,0.15,0.2,0.08)$ \\
$\beta=\left(\beta_{1}, \beta_{2}\right)=(0.15,0.07)$ &
\end{tabular}

The evaluation results are positive and negative factors in a university in Table 2:

Table 2:Factors affecting the results of the evaluation in a university

\begin{tabular}{l|l|l}
\hline \multicolumn{2}{l|}{ Positive factors $\left(x_{k}\right)$} & Negative Factors $\left(y_{k}\right)$ \\
\hline$x_{1}[3,4]$ & $x_{4}[3.5,4.5]$ & $y_{1}[2.5,3.5]$ \\
\hline$x_{2}[4,5]$ & $x_{5}[3.5,4.5]$ & $y_{2}[3.5,4.5]$ \\
\hline$x_{3}[3,4]$ & $x_{6}[3,4]$ & \\
\hline
\end{tabular}

Research institutes for a positive and negative factors evaluation results are shown in Table 3:

Table 3: Evaluation of factors affecting results of a research institutes

\begin{tabular}{l|l|l}
\hline \multicolumn{2}{l|}{ Positive factors $\left(x_{k}\right)$} & Negative Factors $\left(y_{k}\right)$ \\
\hline$x_{1}[1.5,2.5]$ & $x_{4}[2,3]$ & $y_{1}[3,4]$ \\
\hline$x_{2}[1,2]$ & $x_{5}[1.5,2.5]$ & $y_{2}[3.5,4.5]$ \\
\hline$x_{3}[2,3]$ & $x_{6}[1.5,2.5]$ & \\
\hline
\end{tabular}

The evaluation of a university's score: $G_{1}=[3.285,4.285]$

Evaluation of a research institutes Score: $G_{2}=[1.915,2.915]$

For punishment, the development division of the excitation region, the introduction of variable factors $\mathrm{g}, \mathrm{g}$ a major consideration on this project, including the difficulty of the project, project duration, project risk, $g$ smaller the production side easier on Gakken party creates incentives. Due to the different project task difficulty faced by universities and research institutes, its long-term expectation is different,so $g_{1}=-2, g_{2}=1 . \omega=20$, For the evaluation of university get punished development zone incentives are: $[0,1.3],[1.3,3.3],[3.3,5]$, For the evaluation of research institutes penalties development zone incentives are: $[0,1.6],[1.6,3.6],[3.6,5]$. In a university research institutes and a section position belongs to the evaluation value are shown in Figure 3, Figure4. 


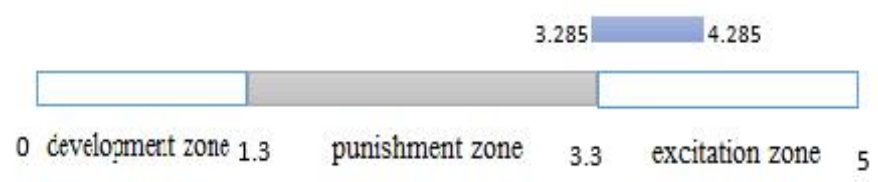

Fig.3:College belongs to an interval evaluation value map

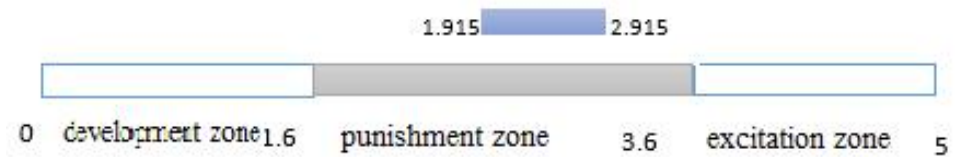

Fig. 4:On a range of research institutes, the evaluation value to your map

Based on Figure 3, Figure 4 analysis, you can easily find the evaluation value of $1.5 \%$ are university development area, $98.5 \%$ belong to the excitation region; evaluation of research institutes value entirely Development Area. Universities determine the probability distribution of benefits of using development function is $1.5 \%$, the probability of using the activation function was $98.5 \%$; the allocation of research institutes to determine the interests of the development function using the probability is $100 \%$.

Shapley value model plus punitive activation function was amended:

$$
V_{1}^{\prime}=\phi_{1}(v)+1.5 \% q(v)+98.5 \% r(v) \quad V_{2}^{\prime}=\phi_{2}(v)+q(v)
$$

Inwhere, $q(v)=1 \% v(s), r(v)=6 \% v(s)$.

It is calculated after correction of the participating members of the Shapley value model for the distribution of benefits:

$$
\phi(v)=(29.37,21.87,18.43)
$$

By contrast Shapley Value distribution model modification before and after analysis, was found on a more balanced distribution of the benefits allocated after a revised gap becomes larger. In the present research cooperation, the credibility is whether a college or project completion, etc. should be better, and played a central role in the future is expected to be long-term cooperation, the distribution of benefits and therefore higher value. Shapley value model has been revised, more in line with the principle of equity, taking into account the influence of the members of the bargaining power, distribution and research interests are in more value.

\subsection{Conclusion and Outlook}

By the above process cases and comparison of the data the following conclusions:

Shapley value model modification and the introduction of interval number variable factor $\mathrm{g}$, to implement different projects in different evaluation criteria, different incentives penalty function, a more reasonable distribution of benefits programs, reflecting the flexibility, efficiency and fairness of the model. Therefore, based on punishment reward function Shapley value model established in this paper, not only to overcome the deficiencies in the Shapley value Cooperative applications, but also takes into account the risk of the project, the duration of cooperation and a series of factors, a more comprehensive and effective, more equitable alliance can be assigned to the interests of research cooperation play a very good reference.

Many directions to be studied in this paper are still corrected Shapley value model requires in-depth analysis and research in determining $\omega$ variables and variable factor $g$ change in terms of the range and influence function; in addition, belong to different intervals to determine the different penalty functions are also incentives require in-depth discussion, so that the whole correction model is more reasonable.

\section{References}

[1] Hu Jintao.Tsinghua University centenary celebration speech on the Assembly [N] .People's Daily,2011-04-25.

[2] Gavirneni S. Benefits of cooperation in a production distribution environment [J].European Journal of Operational Research, 2001 (130) : 612-622. 
[3] Zhuo Xiangzhi, Wang Xu, Wang Zhenfeng. Evolution of Supply Chain Alliance partnership Game studied [J].Computer Engineering and Applications, 2010,46 (1): 208-210.

[4] Shapley L S. Additive and non-additive set function [D]. Princeton University, 1953.

[5] Hart S, et al. Potential, value, and consistency [J]. Econometrica,1987,(55):935-962.

[6] Ying Ma, Zuofeng Gao, Wei Li, Ning Jiang, Lei Guo. The Shapley Value for Stochastic Cooperative Game[J]. Modern Applied Science, 2009, 2 (4) :76-80

[7] $\mathrm{Na} \mathrm{Xu}$, Jason Gu. Improved Weighted Shapley Value Model for the Fourth Party Logistics Supply Chain Coalition [J]. Journal of Control Science and Engineering, 2013, 2013(1):1-5

[8] Haeringer G. A new weight scheme for the Shapley value [J].Mathematical Social Science, 2006,52(1):88-98.

[9] Diaoli Lin, Zhu Guilong, Xu zhi.Based on Alliance benefits distribution mechanism more weight Shapley value. [J].Industrial Engineering and Management, 2011,16 (4): 79-84,91.

[10] O.Palanc1, S.Z.Alparslan Gök, S.Ergün, G.-W.Weber. Cooperative grey games and the grey Shapley value [J]. Optimization, 2015, 64 (8):1657-1668.

[11] Zhang Yanfeng, Liu Yi, Li Heng. Value Creation and Distribution Analysis Strategic Alliance[J] Management Engineering, 2003,17 (2): 20-23.

[12] Dai Jianhua, Xue Hengxin. Dynamic Alliance Based on the Shapley Value Partners business interests allocation policy[J]. China Management Science, 2004,12 (4): 33-36.

[13] Lee, Y.S. The Sustainability of University-Industry Research Collaboration: A Empirical Assessment [J]. The Journal of Technology Transfer, 2000, 25(2):111-133.

[14] Huang Bo, Meng Weidong, Li Yuyu. Based on the best interests of the Cooperative Incentive bilateral distribution[J] Management Sciences, 2011,14 (7): 31-42.

[15] Zhan Qiumei, Zhang Jieyi. Game Analysis of school-enterprise cooperation and innovation on the profit distribution[J]. Research Management, 2010,22 (5): 8-13.

[16] Bao Xinzhong, Wang Daoping. Cooperative Innovation Cost allocation and income distribution Game Analysis [J].Research and Development Management, 2010,22 (5): 75-81.

[17] XG Yue, Y Cao, M McAleer, From Disorder to Order. Theoretical Economics Letters, 2016, 6, 282-288.

[18] Shapley L S. A Value for n-person games [N].Contributions to the Theory of Games, Princeton NJ: Princeton University Press, 1953:307-317.

[19] Xu Shuang Qiu, Hu Shiqing, Cheng Xiyu .Discuss Shapley value characteristics and limitations [J]. Mathematics in time and Theory, 2013,43 (4): 44-52.

[20] Myerson R B. Conference structures and fair allocation rules [J]. International Journal of Game Theory, 1980,9(3):169-182.

[21] Diao lizhu, Zhu jialong, Xu zhi. Based on Alliance benefits distribution mechanism more weight Shapley value [J]. Industrial Engineering and Management, 2011,16 (4): 79-91.

[22] Lin Jian, Zhang Qiang.Fuzzy Shapley value worth having alliance with preference cooperative Games [J]. Journal of Management Systems, 2014,23 (2): 217-223.

[23] Kamijo Y, Kongo T. Axiomatization of the Shapley value using the balanced cycle contributions property [J].Int J Game Theory,2010(39):563-571. 